\title{
Karl Maramorosch and Farida Mahmood (eds): Rearing animal and plant pathogen vectors, 2nd edition
}

\author{
CRC Press, London, 2014, (ISBN: 978-1-4665-9821-8) vi + 337pp
}

\author{
Simon Carpenter ${ }^{1}$
}

Received: 9 February 2015 / Accepted: 29 April 2015 /Published online: 10 May 2015

(C) The Author(s) 2015. This article is published with open access at Springerlink.com

This book is a revised version of Maintenance of Human, Animal and Plant Pathogen Vectors by the same editors, published in 1999. The use of vector colonies is a major component of investigative studies into some of the most damaging diseases of livestock and crops worldwide. These not only allow studies to be carried out throughout the year on organisms that may only be active seasonally, but also enables production of cohorts that are comparable in age and physiological status. This is vital in many areas of research, particularly in studies of transmission which are often heavily influenced by the age and condition of vectors used. The volume consists of collection and rearing guidance for a series of vectors of plant and animal pathogens, written by experts in each field, and includes a wealth of anecdotal information regarding husbandry techniques and troubleshooting of methods. Its main audience will include those involved in technical maintenance of colony lines within academic, governmental and industrial backgrounds.

In terms of relevance to food security, the book covers the production and maintenance of several vector groups that are of considerable importance. These include a detailed chapter on ticks that are responsible for transmission of protozoan parasites such as Babesia among livestock, which inflicts significant economic losses in developing countries. Whiteflies are also examined which not only inflict mechanical damage on a variety of crop species, but also transmit viral diseases. The latter are of particular interest as geminiviruses, causing leaf curl diseases and transmitted by whiteflies can be a major limiting factor in the growth of crops, including cassava in

Simon Carpenter

simon.carpenter@pirbright.ac.uk

1 The Pirbright Institute, Woking, Surrey, UK
Africa. Outbreaks of cassava mosaic disease in East Africa have been particularly devastating, resulting in significant short falls in production in an area where approximately 20 $25 \%$ of the population rely on cassava as a staple crop. Many of the vectors described appear in risk registers in developed countries, which are outside their endemic range, because they are capable of long-distance travel associated with global trade.

This revised version lacks the original chapters on the rearing of Culicoides biting midges and human lice, but adds bed bugs and Psyllids. For the majority of readers, particularly those with specific interests in the veterinary and medical entomology chapters, there is little justification for investing in this version if you already have the original. In most respects this version of the book shares many of the same advantages and disadvantages as the original volume as few revisions have been made to the shared chapters. On the positive side, there is a vast amount of useful and detailed technical information in most of the chapters that will be useful for those both creating and maintaining vector colonies. Prior to the 1999 volume being published, the majority of this information was only available anecdotally or in single chapters, hence the book was a welcome collation of expertise from across the community. This technical information has been updated to a greater or lesser degree across the revised chapters although the basic approaches in most cases have changed little. A major negative aspect to the current volume, however, is the poor quality and reproduction of photographs used to illustrate the book (many of which look extremely dated). The lack of improvement between editions in this area is very disappointing as this was also a major flaw in the original volume and was commented upon in a previous review (Arme 2000).

The organisation of the book follows individual vector groups or species, which has the advantage of making areas relevant to the reader easier to find. One disadvantage of this 
approach for those with a wider interest is that there are significant areas of repetition among chapters. To reduce these, it might have been useful to split general areas such as laboratory design, biosecurity and quality assurance considerations from the rearing chapters. Systematically considering different biosafety levels for containment, as an example, while contrasting current classifications across the world, would greatly assist the reader in understanding key considerations. There is also an absence of discussion of the consequences of genetic modification of colony lines and scalable production, which similarly would have provided a real selling point for this version of the text.

While much of the technical detail contained in the volume remains correct and useful, several chapters do omit important considerations. As an example, systematic molecular typing of field collections prior to or during colonization attempts is not discussed, along with preservation of type specimens. Detailed information on the origin of colonies is often not recorded and this has had significant consequences for later experimentation. Additionally, there is little information regarding issues in colonizing or collecting vectors from other countries, which is commonly attempted across the world. At the very least it should be stated that this process raises a raft of legislative issues not only in terms of biosecurity, but also in biodiversity regulations. Finally, I feel that the book could also be improved by the addition of online resources, perhaps in the form of editable method sheets for production. Given these are now a common feature of textbooks, this would have been a useful and easily updatable forum for the community.

Open Access This article is distributed under the terms of the Creative Commons Attribution 4.0 International License (http:// creativecommons.org/licenses/by/4.0/), which permits unrestricted use, distribution, and reproduction in any medium, provided you give appropriate credit to the original author(s) and the source, provide a link to the Creative Commons license, and indicate if changes were made.

\section{Reference}

Arme, C. (2000). Parasitology, 121, 111-113. 